\title{
PREPARAÇÃO, CARACTERIZAÇÃo E APLICAÇÃo DE FASES ESTACIONÁRIAS TIPO C8 MODIFICADAS POR ÓXIDOS METÁLICOS PARA CROMATOGRAFIA LÍQUIDA
}

\author{
Karen Goraieb*, Maria Izabel M. S. Bueno, Carol H. Collins e Kenneth E. Collins \\ Instituto de Química, Universidade Estadual de Campinas, CP 6154, 13083-970 Campinas - SP, Brasil
}

Recebido em 8/1/13; aceito em 9/5/13; publicado na web em 1/7/13

\begin{abstract}
PREPARATION, CHARACTERIZATION AND APPLICATION OF C8 TYPE STATIONARY PHASES MODIFIED BY METALLIC OXIDES FOR LIQUID CHROMATOGRAPHY. This work describes three C8-stationary phases for high performance liquid chromatography based on silica metallized with $\mathrm{ZrO}_{2}, \mathrm{TiO}_{2}$ or $\mathrm{Al}_{2} \mathrm{O}_{3}$ layers, having poly(methyloctylsiloxane) immobilized onto their surfaces. The stationary phases were characterized using XRF, XAS, FTIR, SEM and elemental analysis to determine the physical characteristics of the oxide and polysiloxane layers formed on the surfaces and chromatographically to evaluate the separation parameters. The results show the changes on the silica surface and allowed proposing a structure for the oxide layer, being observed tetrahedral and octahedral structures, what is completely new in the literature. The formation of a homogeneous layer of metallic oxide $\left(\mathrm{TiO}_{2}\right.$ and $\left.\mathrm{ZrO}_{2}\right)$ was observed on the silica. The $\mathrm{C} 8$-titanized and $\mathrm{C} 8$-aluminized stationary phases presented good chromatographic performances, with good values of asymmetry and efficiency. All stationary phase presented few loss of the polymeric layer after the HPLC, indicating that this layer is well attached on the metalized support.
\end{abstract}

Keywords: C8-stationary phases based on metalized silica; physical and chromatographic characterizations.

\section{INTRODUÇÃO}

A cromatografia líquida de alta eficiência (CLAE) é um método capaz de realizar análises quantitativas de um grande número de compostos presentes em vários tipos de amostras, normalmente em escala de tempo de poucos minutos, com alta resolução, eficiência e detectabilidade. A cromatografia líquida em fase reversa (CLAE-FR) é a modalidade mais utilizada, pois apresenta algumas vantagens, tais como: o uso de fases móveis (FM) menos tóxicas e de menor custo, o estabelecimento mais rápido do equilíbrio da coluna após a mudança da FM, a boa reprodutibilidade dos tempos de retenção, a possibilidade do uso de eluição por gradiente e a grande variedade de fases estacionárias (FE) disponíveis comercialmente. ${ }^{1,2}$ Uma das características mais importantes de uma FE tipo fase reversa é sua polaridade variável, que depende dos grupos quimicamente ligados ou imobilizados sobre a superfície da sílica.

A sílica $\left(\mathrm{SiO}_{2}\right)$ é o suporte mais utilizado em CLAE devido a suas vantagens, como grande área superficial e alta resistência mecânica, sendo o material base para se preparar as diversas FE por ligações químicas ou por imobilização. ${ }^{3}$ Ela apresenta caráter fracamente ácido, que pode ser aumentado pela presença de impurezas ácidas, gerando fenômenos de quimissorção de bases ou reações catalisadas por ácidos nas amostras. ${ }^{1}$

Embora a $\mathrm{SiO}_{2}$ tenha vantagens, a sua maior desvantagem está relacionada ao seu uso limitado a uma faixa de $\mathrm{pH}$ entre 2 e 8 . FE com grupos organossilanos quimicamente ligados à base de $\mathrm{SiO}_{2}$ são instáveis em $\mathrm{pH}$ baixos $(\mathrm{pH}<2)$, com perda do organossilano, enquanto que ligações siloxanos são suscetíveis a quebra quando o $\mathrm{pH}$ é maior que 8. Análises cromatográficas feitas fora da faixa de pH de 2 a 8 farão com que ocorra o desgaste da fase estacionária, provocando a diminuição da sua eficiência. ${ }^{1,4,5}$

A metalização da $\mathrm{SiO}_{2}$ é uma possibilidade para superar as limitações da sílica fora da faixa de $\mathrm{pH}$ recomendada. Baseia-se na preparação de uma ou mais camadas de óxido metálico sobre a superfície da $\mathrm{SiO}_{2}$. Recobrimentos da sílica com óxidos de titânio, ${ }^{6}$

*e-mail: goraieb@gmail.com zircônio ${ }^{7}$ e alumínio ${ }^{8}$ para aplicações em catálise foram descritos na década de 1990. Para que ocorra a reação de metalização, é necessário o uso de um reagente "metalizador" (alcóxido metálico), o qual interagirá com os grupos $\mathrm{SiOH}$ da superfície da sílica, formando ligações $\mathrm{Si}-\mathrm{O}-\mathrm{M}\left(\mathrm{M}=\mathrm{Ti}, \mathrm{Zr}\right.$ ou Al) ${ }^{6-8}$

A estequiometria destas reações (e as monocamadas resultantes de óxido metálico) depende do metal em questão. Portanto, uma comparação cuidadosa das estruturas das camadas obtidas com óxidos de Al, Ti e Zr será importante nas interpretações dos mecanismos cromatográficos destes materiais dependentes da geometria da camada de óxido metálico sobre a sílica, tais como, o modo de como o polímero pode ser ligado ao suporte e qual é o tipo de interação mais provável que pode ocorrer entre o soluto e a fase estacionária. Com relação à camada de óxido metálico sobre a sílica, a literatura mostra que, em média, dois grupos silanóis reagem por molécula de alcóxido de titânio e zircônio, ${ }^{6,7}$ enquanto que, aparentemente, apenas um silanol reage por molécula de alcóxido de alumínio. ${ }^{9}$ Porém não há registro na literatura acerca da geometria destas camadas de óxido colocadas sobre a sílica, sendo este um dos objetivos deste trabalho.

$\mathrm{SiO}_{2}$ metalizadas com óxidos de $\mathrm{Ti}$ ou $\mathrm{Zr}$ vem sendo exploradas como suportes para a preparação de FE com polissiloxanos ${ }^{10-16}$ ou com outros polímeros pré-formados a fim de se melhorar a estabilidade destas fases em meios extremamente ácidos e alcalinos, aumentando a sua faixa de aplicação a valores de $\mathrm{pH}$ menores que 2 e maiores que $8 .{ }^{2}$

Assim, os objetivos deste trabalho foram: (i) caracterizar fisicamente as sílicas metalizadas com a monocamanda de óxido metálico, (ii) determinar as contribuições relativas dos caracteres tetraédrico e octaédrico nas ligações das camadas de óxidos de alumínio, zircônio e titânio na superfície da sílica amorfa e propor uma estrutura geométrica das ligações nestas camadas e (iii) verificar qual fase metalizada com uma camada de polimetiloctilsiloxano apresenta melhor desempenho cromatográfico. Para isso, foi feito uso de diferentes técnicas instrumentais, quais foram: Cromatografia de alta eficiência (CLAE), fluorescência de raios-X (FRX), absorção de raios-X (ARX, usando-se a luz síncrotron como fonte de excitação), análise elementar (AE), espectroscopia de infravermelho com transformada de Fourier (IV) e microscopia eletrônica de varredura (MEV). 


\section{PARTE EXPERIMENTAL}

\section{Materiais}

Os alcóxidos usados como agente metalizador, tetrabutóxido de zircônio, $\left(\mathrm{Zr}(\mathrm{BuO})_{4}, 80\right.$ \% m/v em 1-butanol), tetrabutóxido de titânio $\left(\mathrm{Ti}(\mathrm{BuO})_{4}, \geq 97 \%\right.$ de pureza) e isopropóxido de alumínio ( $\mathrm{Al}\left({ }^{\mathrm{i} P O O}\right)_{3}, \geq 98 \%$ de pureza) foram obtidos da Aldrich (Alemanha). As sílicas utilizadas foram Kromasil (Akzo Nobel, Suécia, de partículas esféricas com $5 \mu \mathrm{m}$ de diâmetro e área superficial de $309 \mathrm{~m}^{2}$ para cada grama de $\mathrm{SiO}_{2}$ ) e Polygosil (Macherey-Nagel, Irlanda, de partículas irregulares de $7 \mu \mathrm{m}$ e área superficial de $303 \mathrm{~m}^{2}$ para cada grama de $\mathrm{SiO}_{2}$ ). Os óxidos usados para a preparação de padrões com as sílicas foram: óxido de titânio (Riedel-de Haën, Alemanha, 99,5\% de pureza), óxido de zircônio (Alfa Aesar, 99,7\% de pureza) e óxido de alumínio (Carlo Erba, Itália, 99,5\% de pureza). O organossilano utilizado foi o polimetiloctilsiloxano, PMOS (da United Chemical Technologies, EUA). Butanol (p.a., Nuclear, Brasil) foi usado para preparar as soluções de tetrabutóxido de zircônio e de titânio. Para a preparação de fase móvel, foram usados metanol grau HPLC (Tedia, EUA) e água Milli-Q, filtrados com filtros de papel quantitativo (Whatman, EUA). Para a preparação da mistura-teste para as análises cromatográficas, foram usados uracil (Aldrich, Alemanha), acetona (p.a., Merck, Brasil), tolueno (p.a., Synth, Brasil), naftaleno (Vetec Química Fina LTDA, Brasil), benzonitrila (p.a., Riedel-de Haën, Alemanha), benzeno (p.a., Synth, Brasil). $\mathrm{HNO}_{3}$ concentrado (Merck, Brasil, $65 \%$ m/v), $\mathrm{CHCl}_{3}$ (p.a., Merck), diclorometano (p.a., Merck, Brasil), etanol (p.a., Synth, Brasil), $n$-hexano (p.a., Nuclear, Brasil) e tolueno foram usados para a preparação das fases estacionárias metalizadas.

\section{Preparação da sílica modificada com $\mathrm{ZrO}_{2}$ ou $\mathrm{TiO}_{2}$}

Os métodos empregados para a zirconização e titanização da superfície da sílica foram desenvolvidos no Laboratório de Pesquisas em Cromatografia, LABCROM (Instituto de Química, UNICAMP, Campinas/SP). Primeiro, secou-se $5 \mathrm{~g}$ de sílica (Kromasil ou Polygosil) a $120{ }^{\circ} \mathrm{C}$ por $24 \mathrm{~h}$. Depois, adicionam-se soluções de $\mathrm{Zr}(\mathrm{OBu})_{4}\left(2,28 \mathrm{~mol} \mathrm{~L}^{-1}\right)$ ou de $\mathrm{Ti}(\mathrm{OBu})_{4}\left(2,45 \mathrm{~mol} \mathrm{~L}^{-1}\right)$ em butanol à de $\mathrm{SiO}_{2}$ seca. Depois, a mistura foi homogeneizada, sonicada (até não haver o aparecimento de bolhas de ar e para garantir que haja boa penetração do alcóxido metálico dentro dos poros da sílica), deixada em banho termostático a $25^{\circ} \mathrm{C}$ durante $6 \mathrm{~h}$ e, por último, centrifugada por $15 \mathrm{~min}$. A sílica com a camada de óxido (de Ti ou $\mathrm{Zr}$ ) foi lavada com porções de $15 \mathrm{~mL}$ de tolueno anidro, hidrolizada com uma solução aquosa de $\mathrm{HNO}_{3} 1,010^{-3} \mathrm{~mol} \mathrm{~L}^{-1} \mathrm{e}$ lavada com água bidestilada. Por último, a sílica metalizada foi secada em estufa a 110 ${ }^{\circ} \mathrm{C}$ em estufa por $12 \mathrm{~h}$.

\section{Preparação da sílica modificada com $\mathrm{Al}_{2} \mathrm{O}_{3}$}

A adição da camada de $\mathrm{Al}_{2} \mathrm{O}_{3}$ sobre a sílica foi realizada pelo método descrito por Gushikem, ${ }^{17} \mathrm{com}$ algumas modificações. Primeiro, $5 \mathrm{~g}$ de sílica (Kromasil ou Polygosil) previamente seca a $150{ }^{\circ} \mathrm{C} \mathrm{em}$ estufa foi imersa numa solução $0,14 \mathrm{~mol} \mathrm{~L}^{-1}$ de isopropóxido de alumínio em tolueno seco. A mistura foi refluxada sob atmosfera inerte de $\mathrm{N}_{2}$ (g), durante $24 \mathrm{~h}$. O sólido resultante foi filtrado, ainda sob atmosfera de $\mathrm{N}_{2}$, em um filtro de Schlenck e lavado com tolueno e etanol. O solvente remanescente foi retirado através do uso de vácuo. A fim de se promover a hidrólise da ligação Al-O- $\mathrm{R}$ remanescente, a sílica aluminizada foi imersa em água bidestilada em meio neutro e deixada em repouso por 4-6 h. A sílica aluminizada foi filtrada, lavada com água bidestilada e, por último, seca a $125^{\circ} \mathrm{C}$.
Preparação da fase estacionária pela imobilização de polimetiloctilsiloxano sobre a sílica metalizada

A adição de polimetiloctilsiloxano, PMOS, foi realizada através do método desenvolvido por Jardim et al. ${ }^{18}$ que consiste numa etapa de secagem da sílica metalizada em estufa a $110^{\circ} \mathrm{C}$, a fim de deixar os grupos silanóis da superfície da sílica livres para reagir. Depois, pesou-se o PMOS de modo a obter uma carga de $50 \% \mathrm{~m} / \mathrm{v}$, dissolvendo-o em diclorometano. Adicionou-se a solução de PMOS a $5 \mathrm{~g}$ de sílica metalizada, agitando-se a mistura lentamente durante $3 \mathrm{~h}$. Logo em seguida, a mistura foi deixada em repouso até a evaporação do solvente. A imobilização do PMOS foi feita via radiação gama ou termicamente, de acordo com o óxido metálico adicionado. Após empacotar a coluna com a sílica metalizada (i.e. rechear a coluna com a FE) já com a camada polimérica, o excesso de PMOS foi removido através de uma etapa de extração com $n$-hexano, numa vazão de 1,0 $\mathrm{mL}$ min $^{-1}$ (esta é a etapa de lavagem ou limpeza da coluna empacotada). Após essa extração, tem-se a fase estacionária $\left(\mathrm{SiO}_{2}\right.$ metalizada com PMOS) pronta para uso cromatográfico.

Com as sílicas zirconizadas e titanizadas, a imobilização do PMOS foi feita utilizando-se raios- $\gamma$, com doses de radiação de 80 kGy e 120 kGy, respectivamente. ${ }^{19,20}$

A imobilização térmica do PMOS sobre a sílica aluminizada foi feita de acordo com o método empregado por Bottoli, ${ }^{21}$ onde a sílica aluminizada com PMOS foi colocada dentro de um reservatório de aço inox e submetido à uma temperatura de $120^{\circ} \mathrm{C}$ por $16 \mathrm{~h}$ na presença de ar.

\section{Preparação de padrões e construção das curvas analíticas}

Os padrões foram confeccionados misturando-se $\mathrm{SiO}_{2}$ (Kromasil ou Polygosil) com $\mathrm{Al}_{2} \mathrm{O}_{3}, \mathrm{ZrO}_{2}$ ou $\mathrm{TiO}_{2}$. Todos os óxidos foram previamente secos em estufa a $120{ }^{\circ} \mathrm{C}$ por $24 \mathrm{~h}$, para a remoção de água fisicamente adsorvida. Cada padrão foi preparado misturando-se proporções pré-definidas do óxido metálico e de $\mathrm{SiO}_{2}$, para obter a concentração de metal desejada.

Para zircônio, foram confeccionados padrões na faixa de 2 a 18 $\%$ de $\mathrm{Zr} \mathrm{m} / \mathrm{m}$, com intervalos de $2 \%$ entre um padrão e outro. Os padrões com Ti englobavam a faixa de 2 a $10 \%$ de Ti m/m, também com intervalos de $2 \%$ entre os padrões. Para o alumínio, foram preparados padrões na faixa de 1 a $5 \%$ de $\mathrm{Al} \mathrm{m} / \mathrm{m}$, com intervalos de 1 $\%$ entre eles. Também havia um padrão de sílica pura representando o "zero" das curvas. Os valores dos padrões para cada metal (Al, $\mathrm{Zr}$ e Ti) foram escolhidos baseados em resultados de recobrimento (em porcentagem de metal por grama de $\mathrm{SiO}_{2}$ ) obtidos anteriormente no LABCROM. $^{9-12}$

As curvas analíticas para $\mathrm{Zr}$ e Ti foram obtidas com a técnica de fluorescência de raios-X (FRX), enquanto para o $\mathrm{Al}$ foi usada a espectrometria de absorção atômica (EAA) em chama.

\section{Caracterização física}

As caracterizações físicas dos suportes e das FE metalizadas utilizaram várias técnicas analíticas, as quais são melhores detalhadas nos tópicos abaixo.

\section{Espectrometria de infravermelho com transformada de Fourier, IV}

As análises por IV foram realizadas num instrumento Bomem $\mathrm{ABB}$, modelo MB 100. As amostras foram pastilhadas com $\mathrm{KBr}$ (aglutinador). Os espectros foram obtidos num intervalo de 4000 a $400 \mathrm{~cm}^{-1}$, com $4 \mathrm{~cm}^{-1}$ de resolução, acumulando 16 varreduras. 


\section{Microscopia eletrônica de varredura, MEV}

Foi realizada em microscópio eletrônico de varredura da marca JEOL T-300 para as amostras de sílicas titanizada e zirconizada, a fim de verificar se há homogeneidade da camada de óxido.

\section{Análise elementar CHN, AE}

Foi feita num equipamento Perkin Elmer, modelo 2400 CHN após cada etapa de preparação da FE (metalização, adição e extração de PMOS) e após a análise cromatográfica.

\section{Fluorescência de raios-X, FRX}

A FRX foi usada para construir as curvas analíticas para $\mathrm{Zr}$ e Ti, no intuito de determinar a concentração destes metais sobre a sílica e, consequentemente, calcular a razão entre o silanol e o metal ( $\mathrm{SiOH} / \mathrm{M}$, onde $\mathrm{M}$ pode ser $\mathrm{Zr}$ ou Ti). Essas análises foram realizadas num espectrômetro de raios-X de energia dispersiva da marca Shimadzu, modelo EDX 700, com tubo de Rh (fonte) e detector semicondutor de $\mathrm{Si}(\mathrm{Li})$. O padrão/amostra foi colocado dentro de uma cela de polietileno com um filme fino de Mylar (poliéster) com 2,5 $\mu \mathrm{m}$ de espessura, bem esticado em sua base para segurar o padrão/amostra. As condições de irradiação foram: $200 \mathrm{~s}$ de irradiação (tempo morto: $25 \%$ ), colimação do feixe de $10 \mathrm{~mm}$, voltagem do tubo de $50 \mathrm{kV}, 100$ $\mu \mathrm{A}$ de corrente elétrica e resolução de $165 \mathrm{eV}$. Os espectros foram registrados de zero a 40,92 keV, com passo de varredura de $0,02 \mathrm{keV}$, em ar. Todas as análises foram realizadas em triplicatas.

\section{Espectroscopia de absorção atômica, EAA}

O teor de alumínio da sílica aluminizada foi determinado por EAA em chama, removendo-se a camada de Al usando-se uma mistura de ácidos concentrados $\left(\mathrm{HCl}: \mathrm{HNO}_{3}, 3: 1 \mathrm{v} / \mathrm{v}\right)$. O Al foi determinado no sobrenadante.

\section{Absorção de raios-X, ARX}

As análises ARX foram realizadas no Laboratório Nacional de Luz Síncrotron, LNLS, em duas estações de análise diferentes, as quais são: a linha de absorção de raios-X duros para titânio e zircônio (por serem átomos de massa média) e a linha de absorção de raios-X moles para alumínio (devido ao seu baixo número atômico), a fim de se determinar o comprimento da ligação M-O e a ordem local do átomo do metal na superfície da sílica. ${ }^{22}$

As amostras de $\mathrm{SiO}_{2}$ zirconizada e seus padrões (óxidos de $\mathrm{Si}$ e $\mathrm{Zr}$ puros) foram pastilhadas com o aglutinante nitreto de boro. A sílica titanizada e de seus padrões $\left(\mathrm{TiO}_{2} \mathrm{e} \mathrm{SiO}_{2}\right)$ foram preparadas na forma de membranas, onde a amostra em pó foi aderida sobre a superfície de celulose da membrana. A amostra/padrão sobre esta membrana foi enviada para análise em suporte próprio do LNLS. Os espectros de absorção de raios-X foram obtidos na linha de absorção de raios-X duros para ambos os metais, com o programa 3-Win DCM. Um monocromador de $\mathrm{Si}(220)$ foi utilizado no caso das amostras com Zr, por ter borda de absorção com valores acima de $8000 \mathrm{eV}$, ou de $\mathrm{Si}(111)$ para as amostras com Ti, pois sua borda de absorção possui energia inferior a $8000 \mathrm{eV}$. Antes das análises, o equipamento foi previamente calibrado com um filtro de zircônio metálico ou com um de titânio metálico.

As amostras de $\mathrm{SiO}_{2}$ aluminizada e seus padrões $\left(\mathrm{Al}_{2} \mathrm{O}_{3}\right.$ e $\left.\mathrm{SiO}_{2}\right)$ foram analisados na linha de absorção de raios-X moles. Para isso, as amostras e seus padrões foram colocados, separadamente, em fitas de carbono dupla face de $2,0 \mathrm{~cm}$ x $0,7 \mathrm{~cm}$ presas em um suporte de amostras, o qual contém também duas placas de cerâmica fluorescente presas, uma de cada lado (em um lado a placa está na parte superior e no outro na inferior), a fim de se localizar o feixe de radiação. Depois que as fitas com as amostras estavam prontas, as amostras foram deixadas sob vácuo durante um período de $6 \mathrm{~h}$ para a total retirada da água adsorvida sobre a superfície das amostras e padrões numa câmara metálica pertencente a própria estação de análises. O uso do programa 3-Win DCM foi necessário para se obter os espectros. A análise de todos os espectros foi realizado pelo programa WinXAS 2.3.

\section{Análises cromatográficas}

As colunas foram preparadas pesando-se $0,8 \mathrm{~g}$ de fase estacionária (FE), que foi suspenso em $8 \mathrm{~mL}$ de clorofórmio numa proporção 10 $\% \mathrm{~m} / \mathrm{v}$ entre a FE e o solvente. Esta suspensão foi agitada por $12 \mathrm{~h}$, para evitar a sedimentação das partículas.

Com o sistema de bomba de enchimento Haskel montado com uma coluna de aço inox sem costura (dimensão: $60 \mathrm{~mm}$ x 3,9 mm) com um filtro de $2 \mu \mathrm{m}$ conectado em sua parte inferior preso ao sistema, transferiu-se a suspensão para dentro do reservatório, completando-se o volume do reservatório com clorofórmio. Após fechar bem o reservatório, o enchimento das colunas foi realizado com 40 MPa de pressão e metanol como solvente propulsor. Após rechear, cada coluna foi submetida a uma etapa de condicionamento com uma FM 70:30 (v/v) MeOH: $\mathrm{H}_{2} \mathrm{O}$, numa vazão de $0,3 \mathrm{~mL} \mathrm{~min}^{-1}$ por 3 horas.

Todos os testes com as FE foram realizados no modo isocrático em um cromatógrafo a líquido composto por uma bomba de alta pressão (Waters 510), um injetor manual Rheodyne com loop de 5 $\mu \mathrm{L}$, um detector UV-visível (Shimadzu, SPD - 10A) com detecção a $254 \mathrm{~nm}$, e software ChromPerfect for Windows 5.5.6.

As FE foram avaliadas com uma mistura-teste constituída por compostos neutros com diferentes polaridades, cuja composição química é a seguinte: uracil, acetona, benzonitrila, benzeno, tolueno e naftaleno, dissolvidos em metanol puro. A FM foi constituída por uma mistura de 60:40 metanol: $\mathrm{H}_{2} \mathrm{O}$ v/v.

\section{RESULTADOS E DISCUSSÃO}

\section{Caracterização física}

\section{Fluorescência de raios-X, FRX}

As intensidades dos picos $\mathrm{K} \alpha$ e $\mathrm{K} \beta$ de FRX são diretamente proporcionais a concentração do metal de interesse. Para a construção da curva analítica de $\mathrm{Zr}$ e de Ti, relacionou-se as intensidades do pico $\mathrm{K} \alpha$ com a concentração do metal, calculando sua média $(n=3)$ para cada padrão. A regressão linear com $\mathrm{SiO}_{2}$ Kromasil para o Zr é representada pela equação: Intensidade ${ }_{\text {picoK } \alpha}=-1.05+1.95 * \% \mathrm{Zr}$, com coeficiente de regressão 0,99 enquanto que a regressão linear para a sílica titanizada, também preparada com sílica Kromasil forneceu a equação: Intensidade ${ }_{\text {picoKo }}=-0.90+1.68 * \% \mathrm{Ti}$, com coeficiente de regressão 0,99. Para a sílica Polygosil, as regressões lineares para o Zr e para o Ti foram, respectivamente: Intensidade $_{\text {picoK } \alpha}=-0.78+$ $1.60 * \% \mathrm{Zr}$ e Intensidade ${ }_{\text {picoK } \alpha}=-1.23+3.15 * \% \mathrm{Ti}$, ambas com coeficiente de regressão 0,99 . Os resultados para as amostras zirconizadas e titanizadas são mostrados na Tabela 1.

Os dados obtidos por FRX permitiram o cálculo da razão $\mathrm{SiOH} / \mathrm{M}$ ( $\mathrm{M}=\mathrm{Zr}$ ou Ti), que indica o número de camadas de óxido metálico sobre a sílica. A literatura ${ }^{6,7}$ cita que, para a obtenção de uma monocamada completa de $\mathrm{TiO}_{2}$ ou de $\mathrm{ZrO}_{2}$, são necessários $2 \mathrm{SiOH}$ por átomo metálico, ou seja, que a relação $\mathrm{SiOH} / \mathrm{M}$ seja igual a 2 para $\mathrm{Zr}$ e Ti. Este valor varia conforme o grau de evolução da monocamada (completa ou não) ou de mais camadas. Se esta razão for maior que 
Tabela 1. Porcentagem de $\mathrm{Zr}$ e de Ti sobre a sílica determinado por XRF (média de triplicatas). As áreas das $\mathrm{SiO}_{2}$ Kromasil e da Polygosil são $309 \mathrm{~m}^{2} \mathrm{~g}^{-1}$ e $303 \mathrm{~m}^{2} \mathrm{~g}^{-1}$, respectivamente. Lote 1: $\mathrm{SiO}_{2}$ Kromasil metalizada. Lote 2: $\mathrm{SiO}_{2}$ Polygosil metalizada

\begin{tabular}{ccccc}
\hline \multicolumn{5}{c}{ Zircônio } \\
\hline \multirow{2}{*}{ Lote } & $\% \mathrm{Zr}$ & $\begin{array}{c}\mu \mathrm{mol} \mathrm{Zr/} \\
\mathrm{g} \mathrm{SiO}_{2}\end{array}$ & $\begin{array}{c}\mu \mathrm{mol} \mathrm{Zr/} \\
\mathrm{m}^{2}\end{array}$ & $\begin{array}{c}\text { Relação } \\
\mathrm{SiOH} / \mathrm{Zr}\end{array}$ \\
\hline 1 & $12,18 \pm 0,05$ & 1353 & 4,38 & 1,74 \\
2 & $9,21 \pm 0,02$ & 1151 & 3,80 & 2,00 \\
\hline \multicolumn{5}{c}{ Titânio } \\
\hline \multirow{2}{*}{ Lote } & $\% \mathrm{Ti}$ & $\mu \mathrm{mol} \mathrm{Ti} /$ & $\mu \mathrm{mol} \mathrm{Ti} /$ & Relação \\
& \multirow{2}{*}{1} & $\mathrm{~g} \mathrm{SiO}_{2}$ & $\mathrm{~m}^{2}$ & $\mathrm{SiOH} / \mathrm{Ti}$ \\
\hline 2 & $6,90 \pm 0,04$ & 1114 & 3,61 & 2,11 \\
\hline
\end{tabular}

* O número de mols de $\mathrm{SiOH}$ por metro quadrado de $\mathrm{SiO}_{2}$ é 7,6 $\mu \mathrm{mol} \mathrm{m} \mathrm{m}^{-2}{ }^{23}$

2, a monocamada estará incompleta. Para SiOH/M menor que 2, tem-se a formação de uma segunda camada. A Tabela 1 mostra que a sílica Kromasil zirconizada (lote 1) apresenta indícios da formação de uma segunda camada, enquanto que a sílica Polygosil zirconizada (lote 2) tem a monocamada completa.

Para os lotes titanizados, observou-se o lote 2 (Polygosil) apresentou um valor para a relação $\mathrm{SiOH} / \mathrm{Ti}$ próxima à sugerida na literatura ${ }^{6}$ para uma monocamada de óxido metálico. Para o lote 1 (Kromasil), esta relação mostrou a formação de uma bicamada de $\mathrm{TiO}_{2}$.

\section{Espectroscopia de absorção atômica, EAA}

Com relação à sílica aluminizada, a proximidade dos números atômicos do $\mathrm{Al}(\mathrm{Z}=13)$ e do $\mathrm{Si}(\mathrm{Z}=14)$ impede a quantificação de $\mathrm{Al}$ por $\mathrm{FRX}$, pois seus picos $\mathrm{K} \alpha$ são muito próximos $(\mathrm{K} \alpha[\mathrm{Si}]=$ $1,74 \mathrm{keV}$ e $\mathrm{K} \alpha[\mathrm{Al}]=1,48 \mathrm{keV}) .{ }^{24}$ Sendo assim, as concentrações de $\mathrm{Al}(\mathrm{em} \%)$ foram determinadas pela técnica de EAA em chama cujos resultados são apresentados na Tabela 2 .

Tabela 2. Porcentagem de Al sobre a sílica determinado por espectrometria de absorção atômica em chama (média de triplicatas). As áreas das $\mathrm{SiO}_{2}$ Kromasil e da Polygosil são $309 \mathrm{~m}^{2} \mathrm{~g}^{-1}$ e $303 \mathrm{~m}^{2} \mathrm{~g}^{-1}$, respectivamente. Lote 1: $\mathrm{SiO}_{2}$ Kromasil aluminizada. Lote 2: $\mathrm{SiO}_{2}$ Polygosil aluminizada

\begin{tabular}{ccccc}
\hline Lote & $\% \mathrm{Al}$ & $\mu \mathrm{mol} \mathrm{Al} / \mathrm{g} \mathrm{SiO}_{2}$ & $\mu \mathrm{mol} \mathrm{Al} / \mathrm{m}^{2}$ & Relação SiOH/Al \\
\hline 1 & 1,19 & 114,33 & 0,37 & 5,21 \\
2 & 1,47 & 139,38 & 0,46 & 4,13 \\
\hline
\end{tabular}

Através da Tabela 2, observa-se a baixa concentração de Al nas amostras de sílica aluminizada. Conseqüentemente, observou-se baixos valores de $\mu \mathrm{mol} / \mathrm{g}$ e $\mu \mathrm{mol} / \mathrm{m}^{2}$ e uma relação $\mathrm{SiOH} / \mathrm{Al}$ bastante alta, indicando que poucos grupos $\mathrm{SiOH}$ da $\mathrm{SiO}_{2}$ reagiram com o $\mathrm{Al}\left({ }^{\mathrm{i}} \mathrm{PrO}\right)_{3}$. Para as sílicas Kromasil e Polygosil, a formação da monocamada de Al necessita de um teor de $7 \%$ de Al, valor muito maior que os apresentados pelos lotes aluminizados. Por isso é necessário mais estudos para a preparação da monocamada de $\mathrm{Al}_{2} \mathrm{O}_{3}$, pesquisando-se outros reagentes metalizadores e variando-se algumas variáveis, tais como temperatura da reação, tempo de reação, se o uso de banho-maria seria mais apropriado para promover a formação da monocamada de $\mathrm{Al}_{2} \mathrm{O}_{3}$, mudar a atmosfera onde ocorre a reação, etc.

\section{Absorção de raios-X, ARX}

As análises de ARX duros mostram, em geral, a presença de duas regiões em seus espectros: a primeira é a região de espectroscopia de alta resolução da borda de absorção (XANES - do inglês, X-ray absorption near edge structure), que vai de poucos $\mathrm{eV}$ antes da borda de absorção até cerca de $50 \mathrm{eV}$ acima da borda; a segunda região, que é a de espectroscopia da estrutura fina de absorção (EXAFS - do inglês, extended $X$-ray absorption fine structure), engloba o intervalo que vai de cerca de 50 eV a 1000 eV após a borda de absorção. Estas regiões são mais conhecidas na literatura ${ }^{24}$ por sua sigla em Inglês, e por isso serão as únicas siglas que ficarão neste idioma. Ambas as regiões foram tratadas com o programa WinXAS 2.3.

A Figura 1 mostra os espectros de absorção de raios-X para a amostra de $\mathrm{ZrO}_{2}$ pura e para as sílicas zirconizadas, observando-se em todos a borda de absorção do $\mathrm{Zr}$, em $18,05 \mathrm{keV}$ na região XANES. Pela presença deste pico nos lotes zirconizados, é possível confirmar a presença do $\mathrm{Zr}$ sobre a sílica.

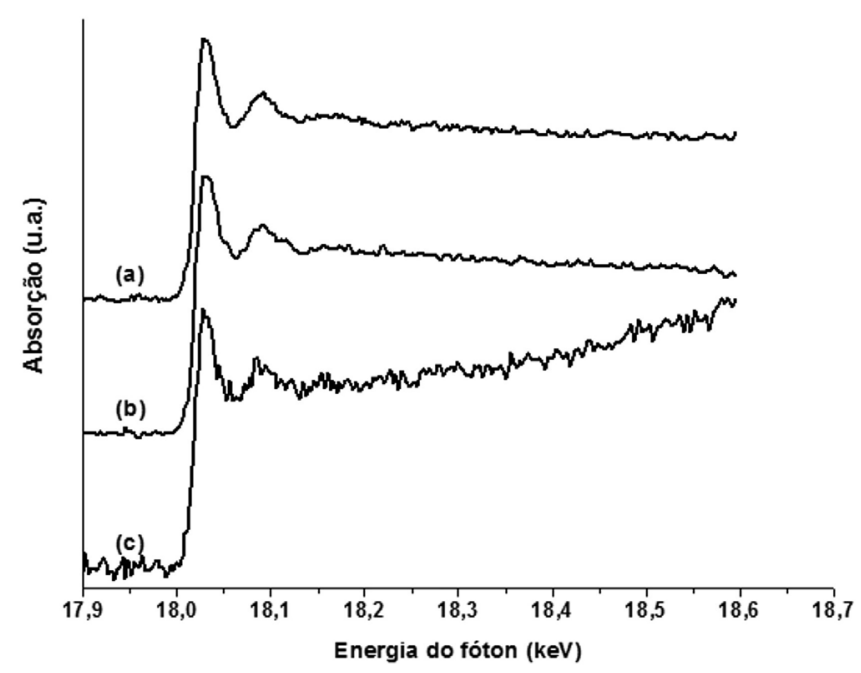

Figura 1. Espectros de ARX para as amostras de sílica zirconizada utilizando (a) sílica Kromasil, (b) sílica Polygosil. (c) Padrão de $\mathrm{ZrO}_{2}$

A região EXAFS foi importante para determinar a distância da ligação $\mathrm{Zr}-\mathrm{O}$ da camada do $\mathrm{ZrO}_{2}$, calcular o número de coordenação médio (NC) do átomo metálico e, conseqüentemente, propor uma geometria para o mesmo e indicar se há ou não $\mathrm{Zr}$ ligado $\mathrm{a} \mathrm{SiO}_{2}$. A zircônia possui três formas diferentes: tetragonal, cúbica e monoclínica, ${ }^{25}$ sendo esta última a forma mais comum, onde o $\mathrm{Zr}$ tem coordenação igual a 7 (forma onde os cálculos de comprimento de ligação e NC foram baseados). ${ }^{26} \mathrm{~A}$ distância média de três dos sete oxigênios ligados ao $\mathrm{Zr}$ é de 0,207 nm, enquanto que a distância média dos outros quatro oxigênios com o $\mathrm{Zr}$ é de $0,221 \mathrm{~nm} \cdot{ }^{27}$ Assim, considerou-se a média destes valores para se calcular o comprimento da ligação $\mathrm{Zr}$-O $(0,215$ $\mathrm{nm}$ ) nas amostras zirconizadas. Na Tabela 3 , podem ser vistos os valores

Tabela 3. Números de coordenação (NC) e comprimento de ligação M-O $\left(\mathrm{M}=\mathrm{Zr}\right.$ ou Ti) para as sílicas metalizadas. Lote $1: \mathrm{SiO}_{2}$ Kromasil metalizada. Lote 2: $\mathrm{SiO}_{2}$ Polygosil metalizada

\begin{tabular}{ccc}
\hline \multicolumn{3}{c}{ Sílica zirconizada } \\
\hline Lote & NC & Distância Zr-O (nm) \\
\hline 1 & $6,01 \pm 0,91$ & $0,212 \pm 0,006$ \\
2 & $5,85 \pm 0,28$ & $0,225 \pm 0,006$ \\
\hline \multicolumn{3}{c}{ Sílica titanizada } \\
\hline Lote & NC & Distância Ti-O $(\mathrm{nm})$ \\
\hline 1 & $5,39 \pm 0,13$ & $0,192 \pm 0,001$ \\
2 & $4,47 \pm 0,78$ & $0,196 \pm 0,001$ \\
\hline
\end{tabular}


das distâncias Zr-O das sílicas Kromasil (lote 1) e Polygosil (lote 2) zirconizadas. Ambos os lotes têm NC muito próximo a 6, indicando a formação de ligação Si-O-Zr. Além disso, pode-se sugerir que a camada de zircônia tem a geometria octaédrica nos dois lotes.

No caso do titânio, primeiro foram obtidos de espectros de absorção de raios-X para os padrões com as duas formas alotrópicas da titânia: rutilo e anatásio, ambas de estrutura octaédrica (NC[Ti] =6). A Figura 2 mostra os espectros destas duas formas, e por isso possibilita observar as diferenças em suas regiões XANES.

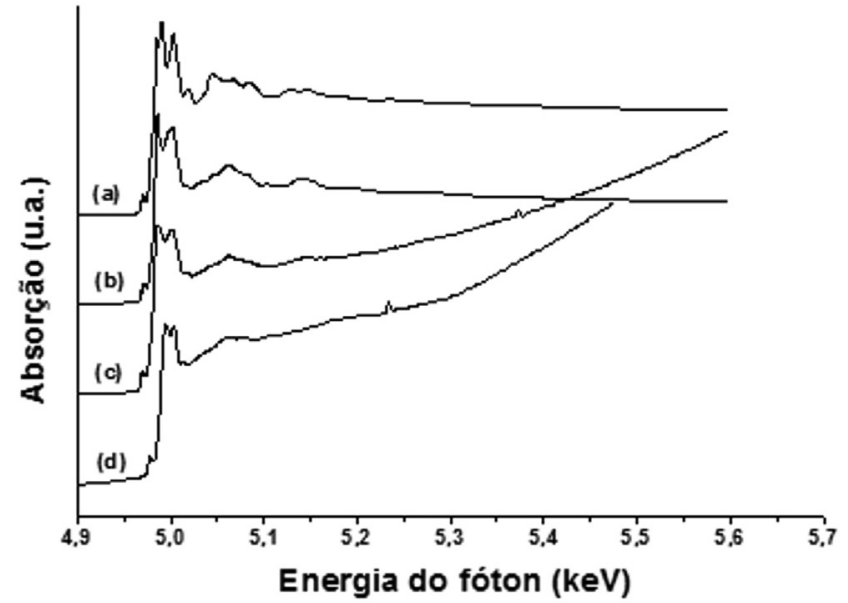

Figura 2. Espectro de ARX para padrões de $\mathrm{TiO}_{2}$ nas formas de (a) rutilo $e$ (b) anatásio e para amostras de silica titanizada dos lotes (c) Kromasil e (d) Polygosil

Através da comparação das amostras de sílica titanizada e dos padrões, observa-se através da região XANES que a camada de titânia sobre a sílica aparentemente está na forma de anatásio (Figura 2) nas sílicas Kromasil (lote 1) e Polygosil (lote 2) titanizadas, o que era esperado, pois esta é a forma mais estável do $\mathrm{TiO}_{2}$ em temperatura ambiente. ${ }^{28}$

Os resultados obtidos a partir da região EXAFS mostram o comprimento da ligação Ti-O é de $0,196 \mathrm{~nm}$ para o lote 1 e de $0,192 \mathrm{~nm}$ para o lote 2 (Tabela 3). O NC dos dois lotes é próximo a 5, também indicando a formação da ligação $\mathrm{Si}$-O-Ti e que a camada de $\mathrm{TiO}_{2}$ ligada a sílica apresenta uma mistura das estruturas tetra e octaédrica.

No caso das amostras aluminizadas, não foi possível obter resultados de NC e nem do comprimento de ligação Al-O via ARX, também pelo fato da sua borda de absorção $K \alpha$ ser muito próxima à do silício $(\mathrm{K} \alpha(\mathrm{Si})=1,84 \mathrm{keV}$ e $\mathrm{K} \alpha(\mathrm{Al})=1,56 \mathrm{keV})$.

A grande inovação trazida pelas análises de ARX foi mostrar a forma geométrica da camada de óxido metálico (de $\mathrm{TiO}_{2}$ e de $\mathrm{ZrO}_{2}$ ) sobre a sílica, o que pode ajudar a elucidar como o PMOS (ou outro polímero) liga-se ao suporte metalizado, além de ajudar a propor como é realizada a interação entre os solutos de uma mistura e a fase estacionária.

\section{Infravermelho com tranformada de Fourier, IV}

Na sílica nua (Figura 3a), silanóis absorvem radiação infravermelha na região de $3000 \mathrm{a} 3900 \mathrm{~cm}^{-1} \cdot{ }^{29}$ Também, sabe-se que o grupo $\mathrm{OH}$ da água adsorvida também absorve nesta região. ${ }^{30}$ Por isso, observa-se uma banda larga nesta região, que representa a soma da absorção do $\mathrm{OH}$ do $\mathrm{SiOH}$ e da água. Outra banda relacionada à água, uma banda de deformação $\mathrm{OH}\left(\delta_{\mathrm{OH}}\right)$ em $1640 \mathrm{~cm}^{-1}$ também aparece no espectro. Na sílica, também se encontram bandas características de quartzo fundido, ${ }^{31,32}$ ou seja, da ligação siloxano $\left(1100 \mathrm{~cm}^{-1}\right.$, $800 \mathrm{~cm}^{-1}$ e $470 \mathrm{~cm}^{-1}$ ). A banda em $1100 \mathrm{~cm}^{-1}$ corresponde ao modo óptico transversal do estiramento assimétrico, $v_{\mathrm{as}}$, da ligação Si-O-Si. A banda em $800 \mathrm{~cm}^{-1}$ está relacionada ao estiramento simétrico, $v_{\mathrm{s}}$, da mesma ligação, enquanto que a banda em $470 \mathrm{~cm}^{-1}$ está relacionada à vibração de deformação da ligação Si-O-Si. Além disso, verifica-se o pico de estiramento do silanol $(\mathrm{SiOH})$ residual, um pico de baixa intensidade situado a $980 \mathrm{~cm}^{-1} \cdot 31,32$

Todos os suportes metalizados (Figura 3b-d) mostraram o quase desaparecimento da banda do $\mathrm{SiOH}$ residual $\left(980 \mathrm{~cm}^{-1}\right)$, sugerindo a reação entre estes $\mathrm{SiOH}$ residuais e o reagente metalizador $\left(\mathrm{Zr}(\mathrm{BuO})_{4}\right.$, $\mathrm{Ti}(\mathrm{BuO})_{4}$ ou $\left.\mathrm{Al}\left({ }^{\mathrm{i}} \mathrm{PrO}\right)_{3}\right)$. Outras bandas são as mesmas apresentadas pela sílica nua, tais como: (a) as bandas de estiramentos (simétrico e assimétrico) e de deformação da ligação $\mathrm{Si}-\mathrm{O}-\mathrm{Si}$, (b) a banda larga relacionada ao grupo $\mathrm{OH}$ (do $\mathrm{SiOH}$ e da $\mathrm{H}_{2} \mathrm{O}$ ) em torno de $3450 \mathrm{~cm}^{-1}$ e (c) a banda da $\mathrm{H}_{2} \mathrm{O}$ adsorvida (ao redor de $1640 \mathrm{~cm}^{-1}$ ). ${ }^{29,30}$

Ao se adicionar o PMOS ao suporte metalizado $\left(\mathrm{com} \mathrm{TiO}_{2}, \mathrm{ZrO}_{2}\right.$ ou $\mathrm{Al}_{2} \mathrm{O}_{3}$ - Figura 4), bandas relacionadas a ligação $\mathrm{C}-\mathrm{H}$ dos grupos $\mathrm{CH}_{2}\left(2930 \mathrm{~cm}^{-1}\right.$ e $\left.2855 \mathrm{~cm}^{-1}\right)$ e $\mathrm{CH}_{3}\left(2965 \mathrm{~cm}^{-1}\right)$ presentes na cadeia carbônica do PMOS. ${ }^{30}$ Para os suportes e as FE preparadas com sílica Polygosil metalizada (com $\mathrm{TiO}_{2}, \mathrm{ZrO}_{2}$ ou $\mathrm{Al}_{2} \mathrm{O}_{3}$ ) com ou sem PMOS não se observam em seus espectros de infravermelho, bandas diferentes daquelas encontradas nos espectros de infravermelho da sílica Kromasil metalizada, e devido a essa semelhança encontrada em espectros, estes não serão mostrados.

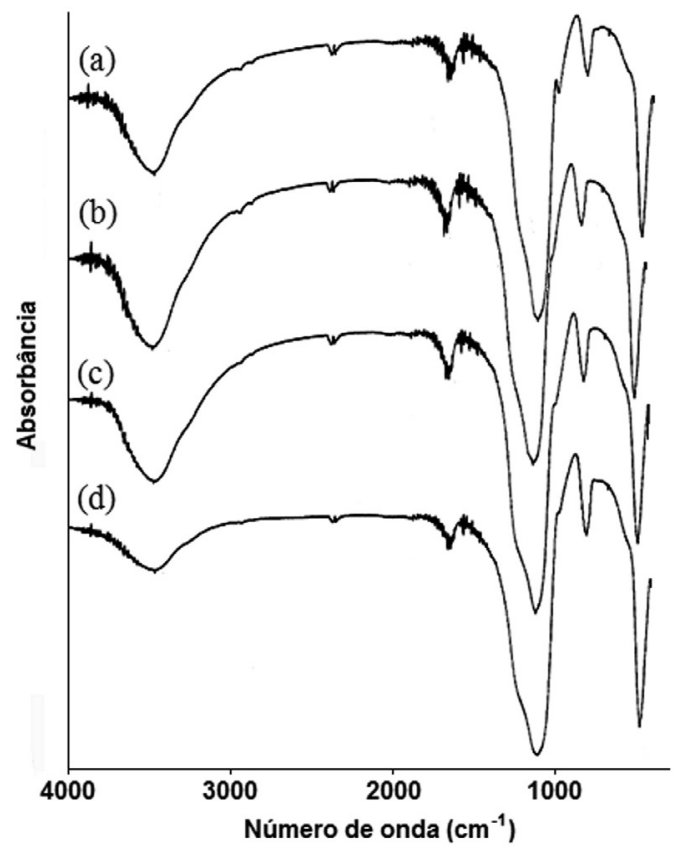

Figura 3. Espectros de IV para a (a) $\mathrm{SiO}_{2}$ Kromasil nua e para sílica (b) zirconizada, (c) titanizada e (d) aluminizada

\section{Microscopia eletrônica de varredura, MEV}

A MEV para as sílicas titanizada e zirconizada mostram um recobrimento bastante homogêneo da monocamada de óxido independente do tipo de sílica utilizado, conforme mostras as imagens da Figura 5. Não foi realizado MEV para as amostras de sílica aluminizada.

\section{Análise elementar CHN, AE}

A porcentagem de carbono obtida pela AE mostrou que as lavagens para remover o excesso de reagente metalizador foram eficientes para os lotes metalizados, pois se observaram resultados de $\%$ C menores que $1,0 \%$, ou seja, menores que o limite de detecção do método para a maioria das sílicas metalizadas (Tabela 4). 


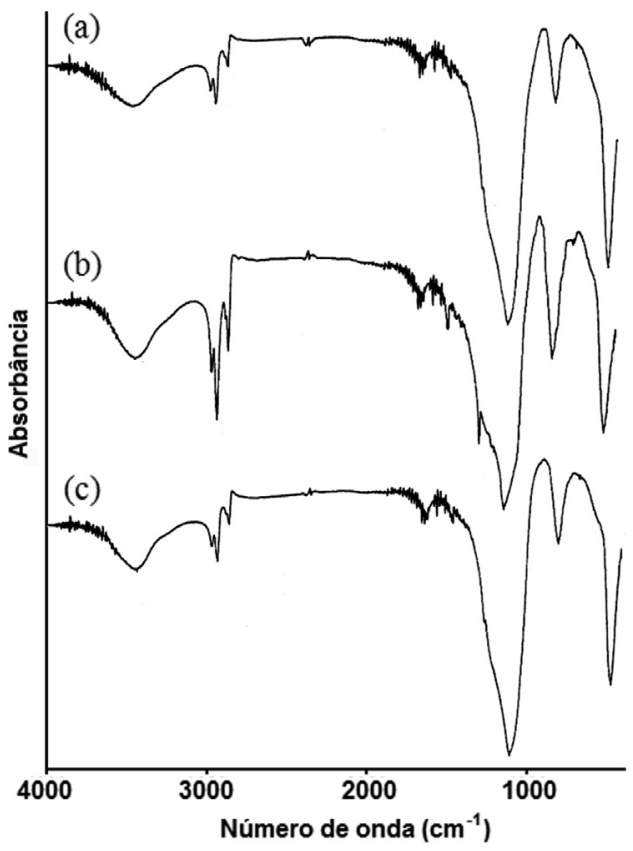

Figura 4. Espectros de IV para as sílicas (a) zirconizada, (b) titanizada e (c) aluminizada com a camada de PMOS

A porcentagem de carbono após imobilização de PMOS e a subseqüente etapa de extração do excesso com $n$-hexano está na faixa de 13 a $18 \%$ de carbono. Após as análises cromatográficas, as fases com sílica zirconizada ou titanizada apresentaram pouca ou nenhuma variação na porcentagem de carbono. Por outro lado, as FE aluminizadas mostraram uma queda bastante pronunciada da $\% \mathrm{C}$, indicando uma maior perda do PMOS, provavelmente relacionada a uma dificuldade em efetuar a imobilização do polissiloxano.

\section{Caracterização cromatográfica}

Testes cromatográficos realizados permitiram obter os parâmetros cromatográficos eficiência $(\mathrm{N})$, fator de retenção (k) e assimetria (As), a fim de avaliar o desempenho de cada FE metalizada. Por convenção, ${ }^{1}$ estes parâmetros são determinados apenas para o composto mais retido, que no presente trabalho é o naftaleno, cujos resultados se encontram na Tabela 5. Os cromatogramas das fases com PMOS são mostrados na Figura 6.

Em geral, a FE preparada com sílica polygosil zirconizada com PMOS apresentam um valor de assimetria adequado, porém, possui um tempo de análise bastante elevado. Ainda, ambos os lotes zirconizados possuem baixa eficiência. As FE preparadas com sílica titanizada ou aluminizada são mais promissoras, com desemprenho relativamente semelhantes, apresentando bons valores de número (a)

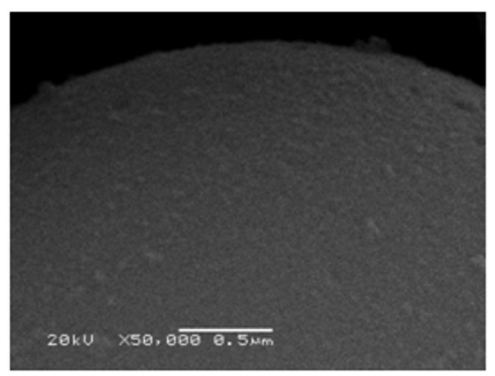

(b)

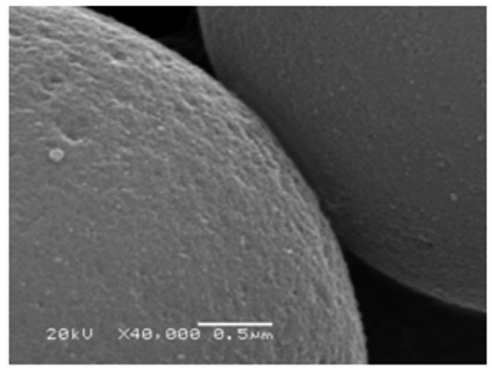

(c)

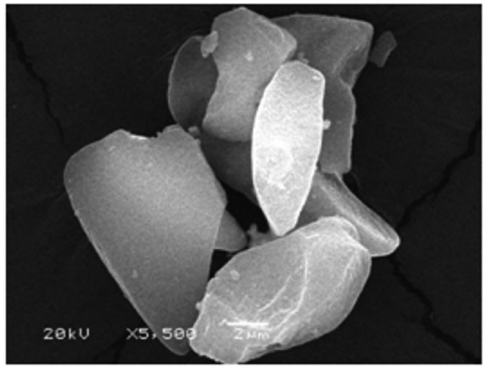

Figura 5. Imagens de MEV para (a) a sílica Kromasil titanizada com aumento de 50000x, (b) a sílica Kromasil zirconizada com ampliação 40000x, e (c) a sílica Polygosil titanizada com aumento de 5500x

de pratos por metro e assimetria (sobretudo para a sílica polygosil metalizada), bem dentro da faixa recomendada na literatura. ${ }^{1}$

Todos os lotes metalizados foram capazes de separar relativamente bem os compostos da mistura-teste. Infelizmente, notou-se que os compostos uracil (pico 1) e acetona (pico 2) co-eluem parcial ou totalmente dependendo da FE empregada. Neste caso, seria necessário o uso de eluição por gradiente (isto é, quando há variação da composição da fase móvel ao longo da corrida) para separar estes dois picos.

Em geral, as FE metalizadas preparadas com $\mathrm{SiO}_{2}$ Kromasil são mais eficientes devido ao fato de suas partículas terem menor diâmetro que as da sílica Polygosil. Isto já era esperado, pois a eficiência de uma coluna está relacionada ao diâmetro das partículas da FE, onde quanto menor é o tamanho das partículas, melhor é a eficiência da coluna. Já as FE metalizadas baseadas em sílica Polygosil apresentam

Tabela 4. Porcentagens de carbono obtidas por análise elementar durante as etapas de preparação das FE metalizadas e após seu uso cromatográfico. Lote 1: $\mathrm{SiO}_{2}$ Kromasil metalizada. Lote 2: $\mathrm{SiO}_{2}$ Polygosil metalizada

\begin{tabular}{|c|c|c|c|c|c|c|}
\hline & \multicolumn{3}{|c|}{ Após a reação de metalização } & \multicolumn{3}{|c|}{ Após imobilização de PMOS e etapa de extração } \\
\hline & $\mathrm{Zr}$ & $\mathrm{Ti}$ & $\mathrm{Al}$ & $\mathrm{Zr}$ & $\mathrm{Ti}$ & $\mathrm{Al}$ \\
\hline Lote 1 & 0,24 & 0,78 & 0,33 & 14,99 & 16,61 & 17,94 \\
\hline Lote 2 & 0,56 & 3,77 & 0,27 & 16,44 & 13,88 & 18,16 \\
\hline \multicolumn{7}{|c|}{ Após análise cromatográfica } \\
\hline & $\mathrm{Zr}$ & $\mathrm{Ti}$ & $\mathrm{Al}$ & & & \\
\hline Lote 1 & 14,59 & 13,98 & 13,18 & & & \\
\hline Lote 2 & 14,14 & 13,57 & 13,47 & & & \\
\hline
\end{tabular}


(a)

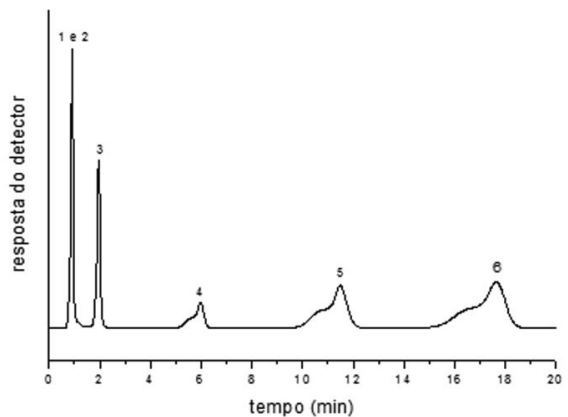

(c)

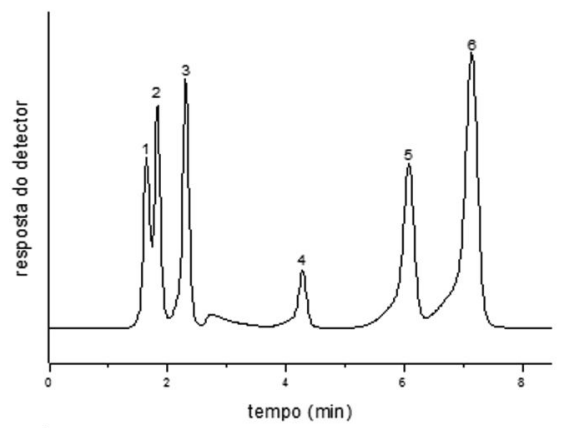

(e)

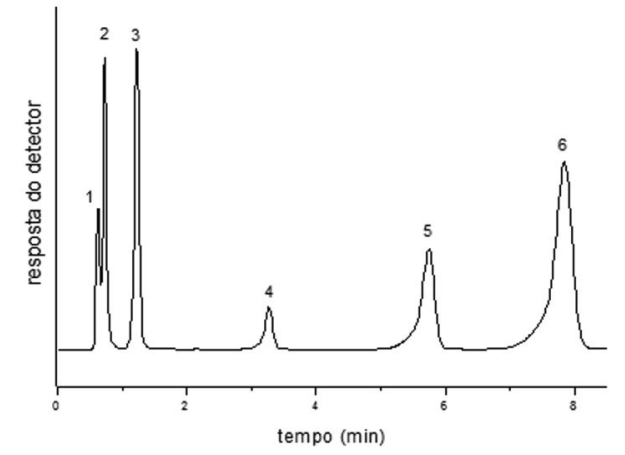

(b)

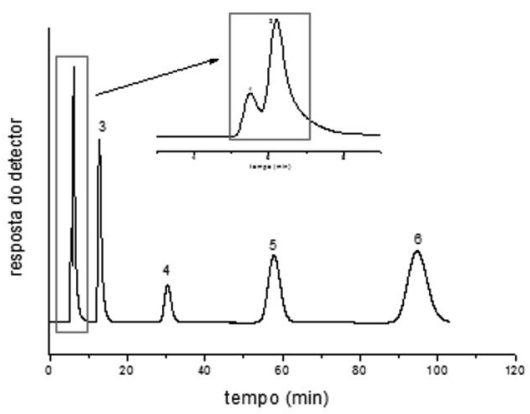

(d)

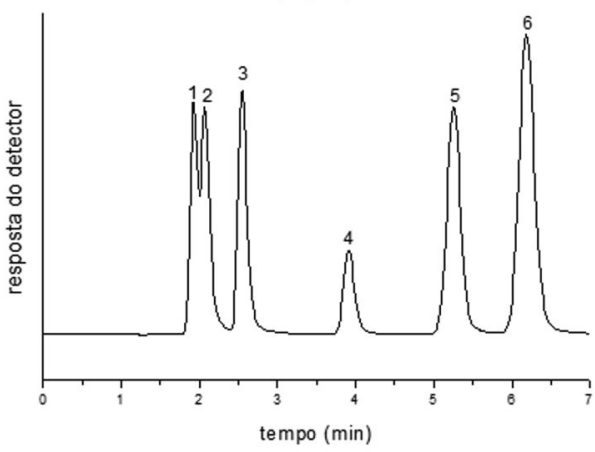

(f)

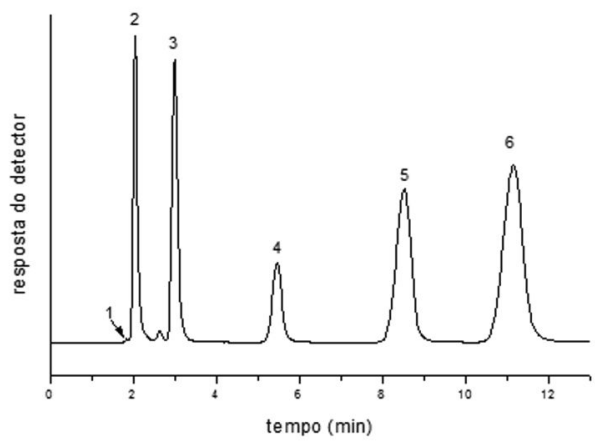

Figura 6. Cromatogramas obtidos para as FE zirconizada com PMOS para os lotes (a) 1 e (b) 2; FE titanizadas para os lotes (a) 1 e (b) 2; FE alumizadas para os lotes (a) 1 e (b) 2. Condições gerais: coluna: $60 \mathrm{~mm} \times 3,9 \mathrm{~mm}$; detecção: $254 \mathrm{~nm}$; FM: 60:40 Metanol:H $\mathrm{H}_{2} \mathrm{O} / \mathrm{v}$. Picos: (1) uracil, (2) acetona, (3) benzonitrila, (4) benzeno, (5) tolueno e (6) naftaleno

picos mais simétricos e melhor separação (Tabela 5 e Figura 6). Uma observação importante é o fato de que todas as FE metalizadas baseadas em sílica Kromasil apresentaram cauda frontal, o que não era esperado pois, este tipo de sílica possui partículas esféricas regulares e apresenta maior pureza que a sílica Polygosil. O que deve ter gerado estas caudas frontais é um provável defeito deste lote de sílica Kromasil, cujas partículas de sílica são mais frágeis que o encontrado normalmente. Isso faz com que durante o enchimento da coluna as partículas de sílica se quebrem devido à pressão exercida pela bomba de enchimento.

\section{CONCLUSÕES}

Os resultados de FRX permitiram determinar as porcentagens de Zr ou de Ti sobre a sílica e, consequentemente, mostraram a formação da monocamada de óxido para os lotes de sílicas zirconizadas ou titanizadas. No caso da determinação da \% Al sobre a $\mathrm{SiO}_{2}$, foi necessário o uso da espectrometria de absorção atômica devido a proximidade dos picos de $\mathrm{Al}$ e Si no FRX (o que consequentemente levaria a interferências espectrais), que indicou uma menor porcentagem de metalização neste caso.

A técnica ARX permitiu obter resultados de comprimento de ligação e de NC para as amostras zirconizadas e titanizadas. Com
Tabela 5. Parâmetros cromatográficos obtidos para o naftaleno usando-se as FE metalizadas com PMOS imobilizado. Lote 1: $\mathrm{SiO}_{2}$ Kromasil metalizada. Lote 2: $\mathrm{SiO}_{2}$ Polygosil metalizada

\begin{tabular}{cccc}
\hline \multicolumn{4}{c}{ FE zirconizada } \\
\hline Lote & $\mathrm{k}$ & $\mathrm{N}($ pratos/m) & As \\
\hline 1 & 16,1 & 37200 & 0,7 \\
2 & 16,2 & 26100 & 1,1 \\
\hline \multicolumn{4}{c}{ FE titanizada } \\
\hline Lote & $\mathrm{k}$ & $\mathrm{N}($ pratos $/ \mathrm{m})$ & $\mathrm{As}$ \\
\hline 1 & 2,8 & 83900 & 0,8 \\
2 & 2,0 & 69600 & 1,1 \\
\hline \multicolumn{5}{c}{$\mathrm{FE}$ aluminizada } \\
\hline Lote & $\mathrm{k}$ & $\mathrm{N}(\mathrm{pratos} / \mathrm{m})$ & $\mathrm{As}$ \\
\hline 1 & 11,5 & 68100 & 0,8 \\
\hline
\end{tabular}

os resultados de NC do metal presente na camada de óxido, foi possível sugerir uma geometria para a mesma, verificando a presença de estruturas tetraédrica (para o Ti) e octédrica (para o $\mathrm{Zr}$ e Ti) e 
indicar a presença de ligações $\mathrm{Si}-\mathrm{O}-\mathrm{M}(\mathrm{M}=\mathrm{Zr}$ ou Ti). Essa foi a grande novidade trazida por este artigo, pois nenhum outro artigo/ revisão descreve a forma como os óxidos metálicos $\left(\mathrm{TiO}_{2}\right.$ e $\left.\mathrm{ZrO}_{2}\right)$ estão presos à sílica.

A espectroscopia de infravermelho foi útil para demonstrar a ocorrência da ligação Si-O-M devido ao fato da diminuição significativa do pico do silanol residual. Após a adição de PMOS aos suportes metalizados, bandas relacionadas a ligações $\mathrm{C}-\mathrm{H}$ presentes na cadeia carbônica do polímero também foram observadas, principalmente as relacionadas aos grupos $\mathrm{CH}_{2} \mathrm{e} \mathrm{CH}_{3}$. A técnica de $\mathrm{MEV}$ mostrou um recobrimento homogêneo das camadas de $\mathrm{ZrO}_{2}$ e TiO${ }_{2}$ sobre a sílica, ou seja, sem pontos de aglomeração destes.

Os testes cromatográficos para as FE metalizadas mostraram que a $\mathrm{SiO}_{2}$ titanizada ou aluminizada com camada de PMOS imobilizada apresentaram bom desempenho cromatográfico, mostrando excelentes valores de assimetria e de eficiência. As FE baseadas em sílica zirconizada apresentaram valores menos animadores. A análise elementar mostrou que há pouca variação na \% $\mathrm{C}$, após a cromatografia com as sílicas titanizadas e zirconizadas, indicando que há pouca perda do PMOS ligado a sílica metalizada após seu uso. Em geral, as FE baseadas em $\mathrm{SiO}_{2}$ Kromasil metalizadas mostraram melhores eficiências (Tabela 5), porém as melhores simetrias foram alcançadas com FE metalizadas baseadas em sílica Polygosil.

Também, ao se comparar os três tipos de fases metalizadas, verificou-se que as sílica titanizada foi a de melhor eficiência. Já a aluminizada, também apresenta valores de eficiência bons, mas ainda precisa ser aperfeiçoada.

Para os suportes metalizados em geral, estudos com outros organossilanos (ex: tipo $\mathrm{C}_{4}, \mathrm{C}_{14}$, grupos fenil) seriam importantes, avaliando outros métodos de imobilização e aplicações cromatográficas. Estudos de aplicação com diversos analitos (fármacos, agrotóxicos) em matrizes mais complexas também seriam de grande valia para a área de separações, aumentando a gama de FE disponíveis para a CLAE.

\section{AGRADECIMENTOS}

Os autores agradecem ao Prof. J. S. Barone pelas análises de FAAS, à FAPESP por um auxílio e uma bolsa concedida e ao LNLS pelo uso das linhas de raios-X duros (projetos XAS 997/01 e XAS 1842/03) e de raios-X moles (projeto SXS 996/01).

\section{REFERÊNCIAS}

1. Collins, C.H.; Braga, G.L.; Bonato, P.S.; Fundamentos de Cromatografia, Editora Unicamp: Campinas, 2006.

2. Tonhi, E.; Collins, K.E.; Jardim, I.C.S.F.; Collins, C.H.; Quim. Nova 2002, 25, 616 .

3. Tonhi, E.; Collins, K.E.; Jardim, I.C.S.F.; Collins, C.H.; J. Chromatogr. A 2006, 1119, 135.

4. Poole, C.F.; Poole, S.K.; Contemporary Practice of Chromatography, Elsevier: Amsterdam, 1991.
5. Snyder, L.R.; Kirkland, J.J.; Glajch J.L.; Practical HPLC Method Development, $2^{\mathrm{a}}$ ed., Wiley: New York, 1997.

6. Gushikem, Y.; Kubota, L.T.; Analyst 1991, 116, 281.

7. Gushikem, Y.; Baccan, N.; Peixoto, C.R.M.; Analyst 1992, 117, 1029.

8. Gushikem, Y.; Castro, S.C.; Prado, L.L.L.; Nascente, P.A.P.; J. Mater. Sci. 2000, 35, 449 .

9. Chiaradia, M.C.; Dissertação de Mestrado, Universidade Estadual de Campinas, Brasil, 2005.

10. Collins, C.H.; Silva, C.R.; Faria, A.M.; Collins, K.E.; Jardim, I.C.S.F.; J. Braz. Chem. Soc. 2009, 20, 604

11. Silva,R.B.; Collins, C.H.; J. Chromatogr. A 1999, 845, 417.

12. Melo, L.F.C.; Jardim, I.C.S.F.; J. Chromatogr. A 1999, 845, 423.

13. Faria, A.M.; Collins, K.E.; Collins, C.H.; J. Chromatogr. A 2006, 1122, 144.

14. Faria, A.M.; Collins, K.E.; Collins, C.H.; J. Chromatogr. A 2007, 1156 , 51.

15. Faria, A.M.; Collins, K.E.; Collins, C.H.; Chromatographia 2008, 67, 357.

16. Faria, A.M.; Silva, C.R.; Collins, C.H.; Jardim, I.C.S.F.; J. Sep. Sci. 2008, 31, 953.

17. Gushikem, Y.; Colloids Surf., A 2001, 178, 135.

18. Jardim, I.C.S.F.; Collins, K.E.; Anazawa, T.A.; J. Chromatogr. A 1999, $849,299$.

19. Melo, L.F.C.; Collins, C.H.; Collins, K.E.; Jardim, I.C.S.F.; J. Chromatogr. A 2000, 869, 129.

20. Silva, R.B.; Collins, K.E.; Collins, C.H.; J. Chromatogr. A 2000, 869, 137.

21. Bottoli, C.B.G.; Tese de Doutorado, Universidade Estadual de Campinas, Brasil, 2002.

22. Gonçalvez, J.E.; Castro, S.C.C.; Ramos, A.Y.; Alves, M.C.M.; Gushikem, Y.; J. Electron Spectrosc. Relat. Phenom. 2001, 114-116, 307.

23. Collins, K.E.; Dimiras, A.B.; de Camargo, V.R.; Collins, C.H.; Microporous Mesoporous Mater. 2006, 89, 246.

24. Jenkins, R.; X-Ray Fluorescence Spectrometry, $2^{\text {a }}$ ed., Wiley: New York, 1999.

25. Raghavan, S.; Wang, H.; Porter, W. D.; Dinwidde, R. B.; Mayo, M. J.; Acta Mater. 2001, 49, 169.

26. Wells, A.F.; Structural Inorganic Chemistry, $5^{\text {a }}$ ed., Clarendon Press: Oxford, 1984.

27. Stevenson, R.; Zirconia and Zirconia Ceramics, $2^{\text {nd }}$ ed., Magnesium Elektron: Twickenham, 1986.

28. Ruiz-López, M.F.; Muñoz-Páez, A.; J. Phys. Condens. Matter 1991, 3 , 8981.

29. Nawrocki, J.; J. Chromatogr. A 1997, 779, 29.

30. Silverstein, R.M.; Bassler, G.C.; Morrill, T.C.; Spectrometric Identification of Organic Compounds, $3^{\mathrm{a}}$ ed., Wiley: New York, 1978.

31. Francisco, M.S.P.; Gushikem, Y.; J. Mater. Chem. 2002, 12, 2552.

32. Wang, S.W.; Mater. Lett. 1996, 28, 436. 